Article

\title{
Analysis of a Set of KDM5C Regulatory Genes Mutated in Neurodevelopmental Disorders Identifies Temporal Coexpression Brain Signatures
}

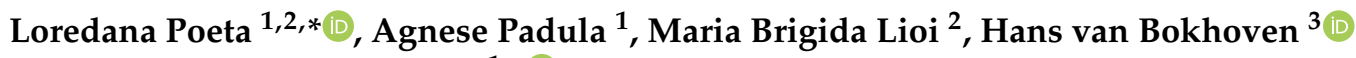 \\ and Maria Giuseppina Miano ${ }^{1, *(\mathbb{D})}$ \\ 1 Institute of Genetics and Biophysics Adriano Buzzati-Traverso, CNR, 80131 Naples, Italy; agne-88@hotmail.it \\ 2 Department of Science, University of Basilicata, 85100 Potenza, Italy; maria.lioi@unibas.it \\ 3 Department of Human Genetics, Donders Institute for Brain, Cognition and Behaviour, Radboudumc, \\ 6525 GA Nijmegen, The Netherlands; Hans.vanBokhoven@radboudumc.nl \\ * Correspondence: poetaloredana@libero.it (L.P.); mariag.miano@igb.cnr.it (M.G.M.); \\ Tel.: +39-(0)-816132261/445 (M.G.M.)
}

\section{check for}

updates

Citation: Poeta, L.; Padula, A.; Lioi, M.B.; van Bokhoven, H.; Miano, M.G. Analysis of a Set of KDM5C

Regulatory Genes Mutated in Neurodevelopmental Disorders Identifies Temporal Coexpression Brain Signatures. Genes 2021, 12, 1088. https://doi.org/10.3390/ genes12071088

Academic Editors: Michele Longo and Tiziana Angrisano

Received: 18 June 2021

Accepted: 15 July 2021

Published: 18 July 2021

Publisher's Note: MDPI stays neutral with regard to jurisdictional claims in published maps and institutional affiliations.

Copyright: (c) 2021 by the authors. Licensee MDPI, Basel, Switzerland. This article is an open access article distributed under the terms and conditions of the Creative Commons Attribution (CC BY) license (https:// creativecommons.org/licenses/by/ $4.0 /)$.
Abstract: Dysregulation of transcriptional pathways is observed in multiple forms of neurodevelopmental disorders (NDDs), such as intellectual disability (ID), epilepsy and autism spectrum disorder (ASD). We previously demonstrated that the NDD genes encoding lysine-specific demethylase 5C (KDM5C) and its transcriptional regulators Aristaless related-homeobox (ARX), PHD Finger Protein 8 (PHF8) and Zinc Finger Protein 711 (ZNF711) are functionally connected. Here, we show their relation to each other with respect to the expression levels in human and mouse datasets and in vivo mouse analysis indicating that the coexpression of these syntenic $\mathrm{X}$-chromosomal genes is temporally regulated in brain areas and cellular sub-types. In co-immunoprecipitation assays, we found that the homeotic transcription factor ARX interacts with the histone demethylase PHF8, indicating that this transcriptional axis is highly intersected. Furthermore, the functional impact of pathogenic mutations of ARX, KDM5C, PHF8 and ZNF711 was tested in lymphoblastoid cell lines (LCLs) derived from children with varying levels of syndromic ID establishing the direct correlation between defects in the KDM5C-H3K4me3 pathway and ID severity. These findings reveal novel insights into epigenetic processes underpinning NDD pathogenesis and provide new avenues for assessing developmental timing and critical windows for potential treatments.

Keywords: neurodevelopmental disorders; X-chromosome; KDM5C; H3K4me3; disease mutations; NDD-related chromatinopathies

\section{Introduction}

Brain development and neuronal differentiation are complex processes involving spatio-temporal changes in chromatin remodeling and transcriptional activity in specific brain regions and neural cell types [1-3]. Alterations in regulatory genes of chromatin remodeling and transcription factors (TFs) cause a huge spectrum of neurodevelopmental disorders (NDDs) [2,4-6]. We recently uncovered genetic and functional relationships within a set of X-linked NDD genes formed by the epigenetic eraser Lysine-Specific Demethylase 5C (KDM5C; MIM:314690) and its upstream regulators, Aristaless-related homeobox (ARX; MIM:300382), PHD Finger protein 8 (PHF8; MIM:300560) and Zinc Finger protein 711 (ZNF711; MIM:314990) [4,5]. KDM5C encodes a highly conserved JmjC-domain protein that reverts the $\mathrm{H} 3 \mathrm{~K} 4 \mathrm{me} 3$ substrate into di- and mono-methylated products, acting as a fine-tuning transcriptional regulator essential for dendritic spine plasticity [7-9]. Mutations in KDM5C were generally found in male children with X-linked syndromic Intellectual Disability (XLID) Claes-Jensen type (MIM:300534), characterized by moderate to severe ID, spasticity, epileptic seizures, short stature, and microcephaly [10-12] or showing a 
developmental delay with or without ASD-like signs [13]. Point mutations or defective gene expression of $K D M 5 C$ may compromise its demethylase activity leading to target gene expression deregulation $[5,14]$. ARX encodes a homeotic bi-functional TF capable of activating or repressing gene transcription [15]. Mutations in ARX have been found in a wide range of NDDs, affecting only male children and including severe cortical malformations such as $\mathrm{X}$-linked Lissencephaly with agenesis of the corpus callosum and ambiguous genitalia (XLAG; MIM:300215); Agenesis of Corpus Callosum (ACC; MIM 300004; also known as Proud syndrome); severe pediatric epilepsy such as Developmental and Epileptic Encephalopathy 1 (DEE1; MIM:308350, also known as West syndrome); and mild cognition diseases including Partington syndrome (MIM 309510), autism, and non-syndromic intellectual disability $[4,16-20]$. Previous studies conducted by us and other groups have shown that XLAG mutations abrogate the transcriptional program controlled by ARX, whereas expansions of the polyA-tracts are hypomorphic mutations with reduced transcriptional activity and binding properties $[4,5,15,21,22]$. PHF8 encodes a histone demethylase whose mutations were found in male children patients with Siderius X-linked mental retardation syndrome (MRXSSD; MIM:300263) characterized by ID, microcephaly, and cleft lip and palate [23-25]. Finally, ZNF711 encodes a TF of the Krüppel C2H2-type zinc-finger protein family that binds mainly to GC-rich CpG island promoters [26]. Variants in ZNF711 have been found in children with non-syndromic ID (MIM:300803) presenting mild to moderate cognition defect and poor speech accompanied by autistic features and mild facial dysmorphisms in some patients [27-29]. Of interest, both KDM5C and its transcriptional regulatory genes are dispersed across the $\mathrm{X}$-chromosome that has been historically targeted for ID studies because variants in these genes give rise to familial recurrence of cognitive defects that affect males more often and more severely than females [30]. Although we have some knowledge of the intersecting regulation of this set of NDD genes, little is currently known about their transcriptional trajectories in the developing brain [5,31]. Consequently, there is no information on their relation to each other with respect to the expression profiles in specific brain areas and/or neuronal cells. To fill this gap, we here report on where and when $K D M 5 C$ and its three regulatory genes are coexpressed. To this end, we integrated data available in public human and mouse databases with in vivo studies performed on embryonic and neonatal mouse brains. Additionally, in NDD patientderived lymphoblastoid cell lines (LCLs) mutated in KDM5C and in its regulatory genes, we also figure out the functional correlation between gene mutation severity and the defect in the KDM5C-H3K4m3 pathway.

\section{Materials and Methods}

\subsection{Animals}

Protocols for animals were approved by the Italian Ministry of Health (DLgs116/92) in accordance with the Institutional Animal Care guidelines of the Institute of Genetics and Biophysics Adriano Buzzati-Traverso.

\subsection{Identification of Cap Analysis of Gene Expression (CAGE) Derived Transcription Start Sites}

Cap Analysis of Gene Expression (CAGE) is a powerful experimental technique for assisting in the identification of transcription start sites (TSSs;) [32,33]. The FANTOM Consortium 2 has extensively applied CAGE on hundreds of tissues and cell lines of humans and mice and characterized the regulatory mechanisms of gene expression. Analysis of FANTOM5 database was performed using Zenbu browser genomic data visualization tools (https: / fantom.gsc.riken.jp/zenbu/ accessed on 1 May 2021). Robust CAGEs defined by DPI clustering falling inside the RefSeq regions of KDM5C, ARX, PHF8 and ZNF711 genes were selected. The expression values are shown in Tags Per Million (TPM) calculated on a per-library total expression. TPM values were obtained for each CAGE using the FANTOM5 expression dataset for tissues, cell lines, and primary cells in human and mouse (Supplementary Tables S1 and S2). 


\subsection{Cell Lines, Transient Transfection, and Luciferase Assay}

SH-SY5Y cell line was maintained in Dulbecco's modified Eagle's medium (Life Technologies ${ }^{\mathrm{TM}}$ Scotland, Swindon, UK) supplemented with 10\% fetal bovine serum (FBS, Life Technologies ${ }^{\mathrm{TM}}$ Bleiswijk, Bleiswijk, The Netherlands), 100 units/mL penicillin and $100 \mathrm{mg} / \mathrm{mL}$ streptomycin (Gibco BRL, Paisley, Scotland). For lymphoblastoid cell line (LCL) establishment, peripheral blood mononuclear cells (PBMCs) were isolated and cultured in RPMI 1640 (Life Technologies, Swindon, UK) using standard methods. All cell lines were tested regularly for mycoplasma contamination.

Transient transfections were performed according to standard methods [4]. The reporter activities were measured using the Dual-Luciferase Reporter Assay System (Promega Corporation Madison, Madison, WI, USA). Each assay was performed in duplicate in three independent experiments and the resulting firefly luciferase values were normalized using the renilla values.

\subsection{Plasmids}

The wild-type ARX expression plasmid and its mutants c.298_330dup33 [p.Ala105 _Ala115] and c.429_452dup24 [p.Ala148_Ala155] have been previously described [4]. The pCMV-HA tagged PHF8, pCMV-Myc tagged PHF8, and pCMV-Myc tagged ZNF711 constructs expressing the human cDNA of PHF8 and ZNF711 are a gift from K. Helin (Center for Epigenetics Research at Memorial Sloan Kettering Cancer Center \& University of Copenhagen, København, Denmark) described elsewhere [34]. Immunoblot analysis of the transfected cell lysates was performed to prove that the overexpressed protein levels were present in all cell lines.

\subsection{Western Blotting and Protein Interaction Assays}

Protein extracts from mammalian cells and tissues were prepared and separated following standard methods [5]. After the blocking of nonspecific binding sites on the membranes with $5 \%$ non-fat milk, the membranes were incubated with specific antibodies. The following antibodies were used: anti-ARX ( $2 \mu \mathrm{g} / \mathrm{mL}$ final; H70 sc-98895, Santa Cruz Biotechnology, Inc. Europe, Heidelberg, Germany), anti-KDM5C (1:1000, H-99 sc-98701, Santa Cruz Biotechnology, Inc. Europe, Heidelberg, Germany), anti-H3K4me3 (1:5000, AB8580, Abcam Cambridge, Waltham, MA, USA), anti-PHF8 (1:500, Millipore 09-868, Merck, Darmstadt, Germany), anti-SYN1 (1:300, Calbiochem 574777, Merck, Darmstadt, Germany), anti-ZNF711 (1:1000, AB-187324, Abcam Cambridge, Waltham, MA, USA). Anti- $\beta$-actin (1:3000, C4 sc-47778, Santa Cruz Biotechnology, Inc. Europe, Heidelberg, Germany) was used as loading controls. The signals were detected with an enhanced chemiluminescence kit (Amersham Biosciences, British Isles, Buckinghamshire, UK) and the films were processed for densitometric scanning. The protein interaction studies were performed using Dynabeads ${ }^{\circledR}$ Protein G Immunoprecipitation Kit (Thermo Fisher Scientific, Waltham, MA, USA) following the manufacturer's instructions. For the immunoprecipitation assays of the endogenous PHF8 and ARX, total lysates from SH-SY5Y cells were used. After SDS-page, Western blotting was performed using anti-ARX (1:500, H70 sc-98895, Santa Cruz Biotechnology, Inc. Europe, Heidelberg, Germany) and anti-PHF8 (1:500, Millipore 09-868, Merck, Darmstadt, Germany) antibodies. For the co-immunoprecipitation assays, the SH-SY5Y cells were transfected with the expression vectors pCMV-HA-PHF8, pCMV-Myc-ZNF711 and pCMV-Myc-ARX. The antibodies used were the anti-cMyc antibody (1:5000, Clone 9E10 M4439, Sigma, Saint Louis, MO, USA) and anti-HA antibody (1:5000, AB-9110, Abcam Cambridge, Waltham, MA, USA). The flow-through derived from each experiment was tested as a negative control of the binding specificity. 


\subsection{RNA Extraction and Real-Time Polymerase Chain Reaction}

Total RNA extraction was carried out with TRIzol procedure (Life Technologies, Scotland, Swindo, UK). Reverse transcription was performed with a QuantiTect Reverse Transcription kit (Qiagen, Hilden, Germany) and steady-state mRNA abundance was determined using the Power SYBR Green PCR Master Mix (Applied Biosystems, Foster City, CA, USA) on the 7900HT Fast Real-Time PCR System (Applied Biosystems, Foster City, CA, USA). Sequences of oligonucleotide primers used in qRT-PCR are reported in Supplementary Table S3. Each experimental assay was performed in triplicate in three independent experiments.

\subsection{Statistical Analysis}

The statistical analyses were performed with the GraphPad Prism 4 software (GraphPad Software) to calculate the significance of the differences among each sample against the control. One-way ANOVA test with Bonferroni correction or the Student's $t$-test was used. The standard error of the mean was used to estimate variation within a single assay.

\section{Results}

\subsection{Spatiotemporal Expression Patterns of KDM5C and Its Regulatory Genes in Human and Mouse Brain}

To test whether KDM5C and its three regulator genes are coexpressed across embryonic and adult brain tissues, we used public resources of the Human Brain Transcriptome Atlas available at https://hbatlas.org/hbtd/basicSearch.pl (accessed on 2 April 2021) [35]. In six brain areas-neocortex (NCX), hippocampal formation (HIP), amygdala (AMY), striatum (STR), midbrain (MB), and cerebellum (CBC) - we observed the high expression levels of the two erasers KDM5C and PHF8 in the early days of neuronal development (period 1: embryonic development $4<$ Age $<8$ PCW corresponding to the first lamination of the cerebral wall; Figure 1A,B). Then, we observed a progressively declined decrease with age until the birth (period 7: late fetal development $24<$ Age $<38$ PCW characterized by the transition from the typical fetal lamination pattern into an adult-like lamination pattern of the cerebral wall; Figure 1A,B). The transcription factor genes ARX and ZNF711 showed a progressive increase in the early stages of development until period 3 (early fetal development $10<$ Age $<13$ PCW characterized by the presence of the bilaminate cortical plate) and period 4 (early mid-fetal development $13<$ Age $<16$ PCW characterized by the consolidation of the cortical plate and the formation of a large, synapse-rich subplate zone). In particular, $A R X$ showed an oscillator expression profile exhibiting an increase in STR (period 3; Figure 1A,B), followed by a change in the opposite direction, with a progressive decreasing trend in the remaining five brain areas, from the early days of neuronal development to the birth (Figure 1A,B). Regarding ZNF711, its gene expression increased gradually from the first days of neuronal development with a peak at the middle of the embryonic phase and then gradually decreased until birth (period 3; Figure 1A,B). Additional evidence for their coexpression during neuronal differentiation derives from transcriptomics resources of human induced pluripotent stem cells (hiPSC) differentiated into neurons (Supplementary Figure S1; http:/ / stemcell.libd.org/scb / accessed on 1 August 2021). Furthermore, in the postnatal lifespan, we observed a similar trend for each NDD gene among the regional expression profiles in human brain (Figure 1A). In addition, we analyzed the expression profile of the X-linked epilepsy gene Synapsin I (SYN1; MIM:313440; [36]), a repressed downstream target of $K D M 5 C$ encoding a neuronal phosphoprotein involved in synaptic neurotransmission [5]. In agreement with this regulation pattern, we observed a gradual increase of SYN1 in each of the six brain areas, from the early days of conception to the birth (Figure 1A). 
A

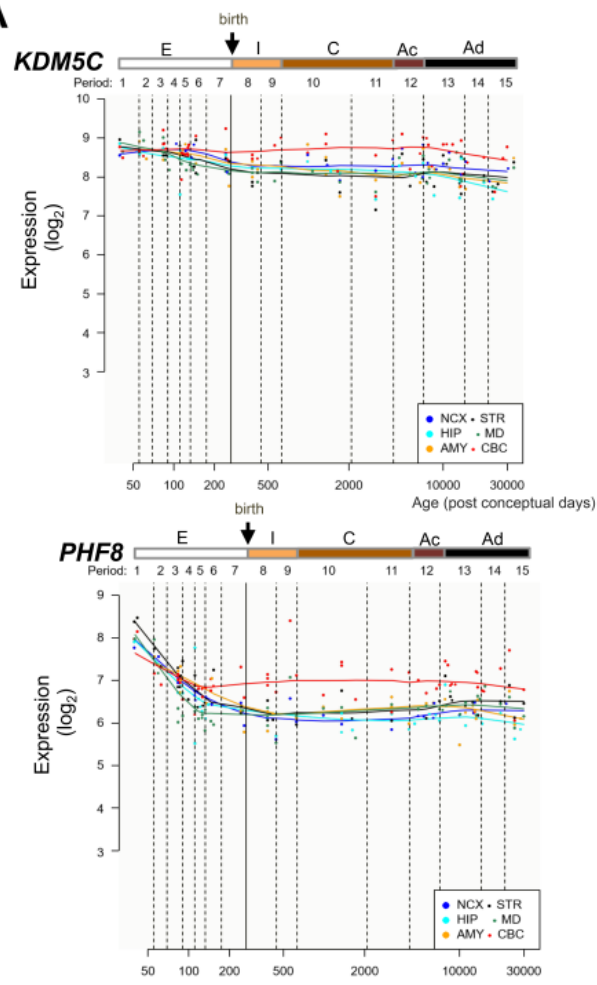

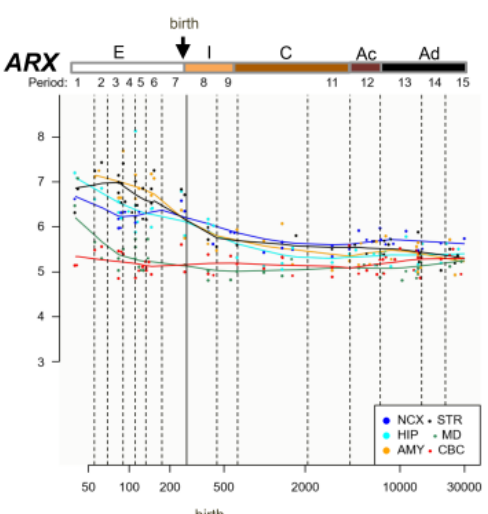

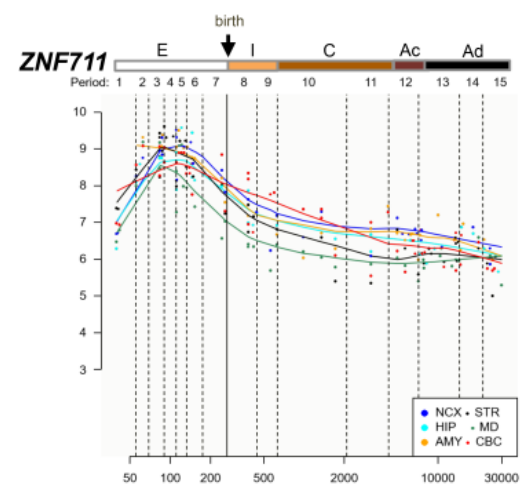

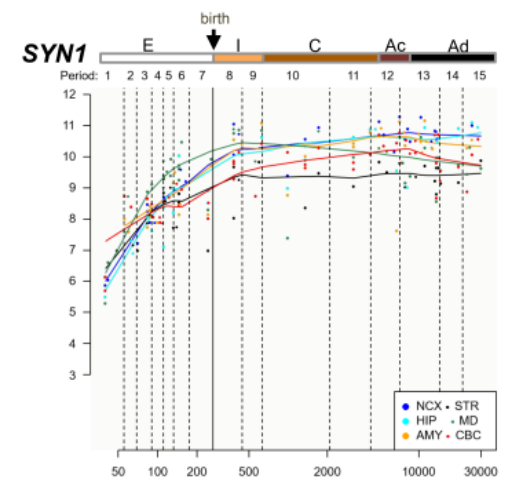

B

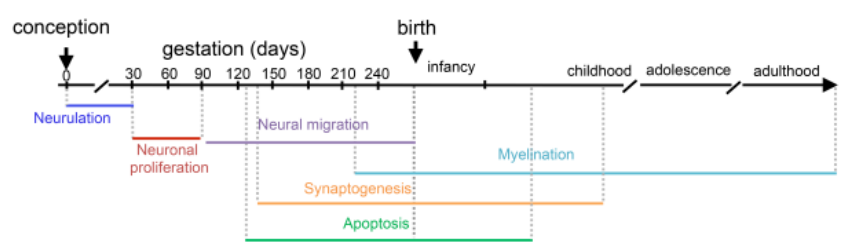

Figure 1. Expression profiles of KDM5C, ARX, PHF8, and ZNF711 during development and in the adult human brain. (A) Data from Human Brain Transcriptome Atlas are shown in RPKM. Original images can be accessed freely at https: // hbatlas.org/hbtd/basicSearch.pl (accessed on 2 April 2021). Period 1, $4<$ Age $<8$ postconceptional weeks (PCW); Period 2, 8 < Age < 10 PCW; Period 3, $10<$ Age < 13 PCW; Period 4, $13<$ Age < 16 PCW; Period 5, $16<$ Age < 19 PCW; Period 6, $19<$ Age < 24 PCW; Period 7, $24<$ Age < 38 PCW; Period 8, Neonatal and early infancy, birth $\leq$ Age $<6$ postnatal months; Period 9, Late infancy, $6<$ Age $<12$ postnatal months; Period 10, Early childhood, $1<$ Age $<6$ years; Period 11, Middle and late childhood, $6<$ Age $<12$ years; Period 12, Adolescence, $12<$ Age $<20$ years; Period 13, Young adulthood, $20<$ Age < 40 years; Period 14, Middle adulthood, $40<$ Age < 60 years; Period 15, Late adulthood, $60+$ years [35]. Embryonic development (E), from 1 to 7; birth and infancy (I), from 8 to 9; childhood (C), from 10 to 11; adolescence (Ac), 12; adulthood (Ad), from 13 to 15. Brain regions: neocortex (NCX), hippocampal formation (HIP), amygdala (AMY), striatum (STR), midbrain (MB), cerebellum (CBC). (B) A glimpse of developmental-stage-specific chronology for neurogenesis (from the embryonic life to the adult stage in human).

Next, we assessed the dynamics of this regulatory axis during mouse embryonic development (at E12.5, E14.5, and E18.5) and during post-natal life (at birth, P0, and P10; Figure 2A-C). By real-time PCR and western blot analysis, we observed that $K d m 5 c$ (GenBank NM_145997.2), Arx (GenBank: BC052033.1), Znf711 (in mouse named Zfp711, GenBank: NM_177747.3), and Phf8 (GenBank: BC138899.1) are all highly expressed at the beginning of neurogenesis (between E12.5 and E14.5), both at mRNA and protein levels (Figure 2A-C). Then, the level of $K d m 5 c / K D M 5 C$ gradually decreases during synapse formation (from E18.5 to P10; Figure 2A-C), as well as Zfp711/ZFP711 and Phf8/PHF8. Regarding the level of $A r x / A R X$, we observed a gradual decrease from E18.5 to P10 as mRNA and a similar protein band from E12.5 to P0 that declines after birth (Figure 2A,B). Taken together, these data provide evidence that the expression patterns of $K d m 5 c$ and 
its regulatory genes overlap prenatally and disappear post-natally. Moreover, in situ hybridization images obtained from the Allen Institute for Brain Science showed that in adult male mice, $K d m 5 c$, Arx, Phf8, and Zfp 711 are coexpressed mainly in the hippocampus and olfactory bulbs (Supplementary Figure S2; https:/ / mouse.brain-map.org/ accessed on 8 April 2021). Noteworthily, the hippocampus and olfactory bulbs are the sites of adult neurogenesis [37]. We also tested the level of Syn1, which transcriptional expression is known to be repressed by KDM5C [38,39]. As expected, the SYN1 protein displays a later onset (E18.5) followed by a gradual increase after birth, which inversely mirrors the decrease of KDM5C (Figure 2B). In conclusion, these findings highlight that in both human and mouse, the $K D M 5 C / K d m 5 c$ transcriptional regulation is organized into coexpression modules operating at specific timing of neurogenesis.
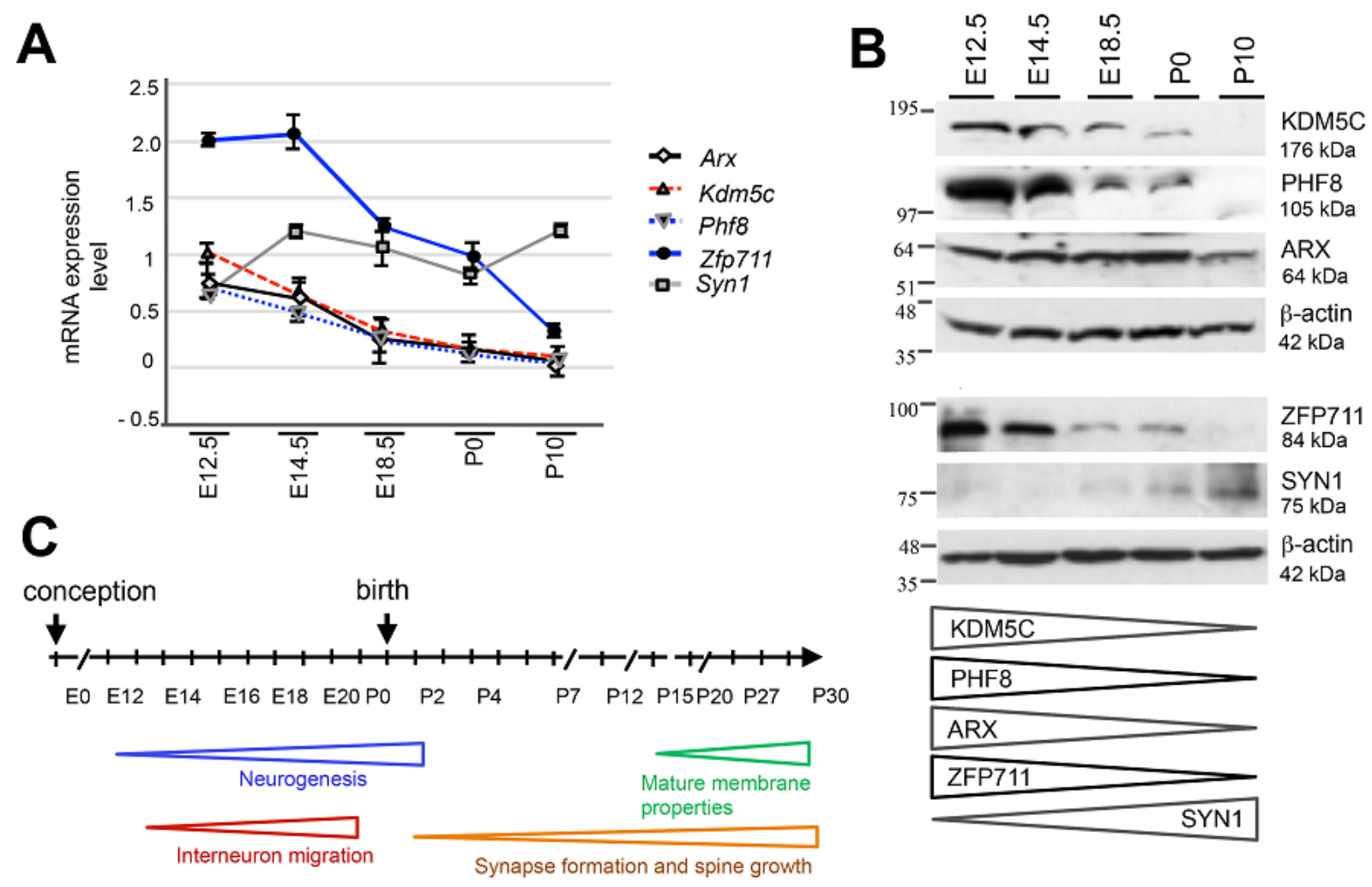

Figure 2. Expression profiles of $K d m 5 c, A r x, P h f 8$ and $Z f p 711$ in embryonic and neonatal mouse brain. Representative quantitative real-time PCR (A) and western blot analysis (B) of Arx/ARX, Phf8/PHF8, Kdm5c/KDM5C, Syn1/SYN1 and Zfp711/ZFP711, throughout prenatal and postnatal stages in the whole WT murine brain. The transcript analysis was performed in triplicate and the samples were normalized to $18 S$. The bars indicate the mean \pm standard error of three independent experiments. The Western blotting experiment was repeated three times. The beta-actin antibody ( $\beta$-actin) was used as a loading control. (C) Schematic representation of the neuronal developmental time line (days) from conception to birth and from birth until 30 days after birth (P30).

\subsection{CAGE-Defined TSSs of KDM5C and Its Regulatory Genes in Human and Mouse Brain}

We analyzed data of promoter level expression across the transcriptome of different brain areas and neuronal cells from the FANTOM 5 project database [40] (https: / / fantom. gsc.riken.jp / zenbu / accessed on 1 May 2021). By using the Cap Analysis of Gene Expression method (CAGE), we identified the predominantly used transcription start sites (TSSs) for the four genes KDM5C, ARX, PHF8, and ZNF711 (Supplementary Figure S3 and Table S1). Specifically, we established that in human brain, the highest used TSS for KDM5C is p1@KDM5C located at chrX:53211978-53263129(-); for ARX, it is p1@ARX located at chrX: 25034067-25034088(-); for PHF8, we identified p1@PHF8, located at chrX:54070731-54070781(-), and p2@PHF8 located at chrX:54070879-54070917(-); and for ZNF711, it is p1@ZNF711 lo- 
cated at chrX:84498989-84499003(+) (Supplementary Figure S3A-C and Table S1). In mouse brain, the highest used Kdm5c TSS is p1@Kdm5c located at chrX:148667771-148667803(+); for Arx, it is p1@Arx located at chrX:90531838-90531861(+); for Phf8, it is p1@Phf8, located at chrX:147955689-147955749(+), and p2@Phf8 located at chrX:147955198-147955224(+); and for Zfp711, it is p1@Zfp711 located at chrX:109714331-109714347(+) (Supplementary Figure S3A$\mathrm{C}$ and Table S1). Successively, we extracted the expression value for each TSS measured as tag per million (TPM) (Figure 3A-C). We generated the heatmap of human promoters using the value of TPM expression (Figure 3A; Supplementary Table S2). We found a strong expression of p1@KDM5C, p1@ARX, p1@PHF8, p2@PHF8 and p1@ZNF711 (expression module embryonic-e) in fetal sub-areas of the cortex (Figure 3A, panel $e$ ). In line with these evidences, the TSSs of the four NDD genes are expressed in a fetal brain pool (59 samples between 20-33 weeks; CNhs1086459_from p1@ARX, the most expressed, to p1@PHF8, the least expressed (Figure 3B; Supplementary Table S2). Moving to neonatal and adult brain samples, we found several changes with respect to fetal profiles (Figure 3A, panels $n$ and $a$ ). In the neonatal brain samples, the coexpression of the TSSs is still present but less strong (expression module neonatal- $n$ ). Instead, in the adult samples, we observed a robust coexpression only of p1@KDM5C and p2@PHF8 since the levels of p1@ARX and p1@ZNF711 are severely reduced in most of the adult samples (expression module adult- $a$; Figure 3A, panel $a$ ). More interestingly, primary neuronal cells showed a weak coexpression of p1@KDM5C,p1@ARX, p2@PHF8, and p1@ZNF711 (expression module-neuron cell-c; Figure 3A), while astrocytes showed two distinctive coexpression profiles: p1@KDM5C, p1@PHF8, and p2@PHF8 in cerebellum (expression module-astrocyte cerebellum-c; Figure 3A); and p1@KDM5C, p1@ARX, p1@PHF8, p2@PHF8, and p1@ZNF711 (expression module-astrocyte cortex-c; Figure 3A). Additionally, in neuronally differentiated induced pluripotent stem cells (iPS), we observed that p1@KDM5C, p1@ARX, p2@PHF8, and p1@ZNF711 are expressed from the induction of neuro-ectodermal cells (day 6) to the generation of early neuronal progenitors (day 18). They showed an oscillatory trend with a peak of p1@ARX and p2@PHF8 at day6 and of p1@KDM5C and p1@ZNF711 at day 12 corresponding to the generation of self-renewing multipotent neural stem cells (Figure 3C).

In neonatal mice, we found a strong expression of p1@Kdm5c, p1@Arx, p2@Phf8, and p1@Zfp711 in corpus striatum (STR) and hippocampus (HIP), both at age N10 (expression module STR/HIP- $n$ ) and in visual cortex at age N15 (Figure 3D and Supplementary Table S2). In adult mice, we noted a strong expression of all TSSs only in the olfactory area (expression module OP- $a$ ). In the remaining areas, a strong decrease in p1@Arx was observed in cortex (CX), HIP, and cerebellum (CB) with respect to OP (expression module CX/HIP/CB- $a$; Figure 3D). Moving to primary cells, we found strong expression of all TSSs in neurons isolated from HIP and STR (coexpression module HIP/STR-c; Figure 3D). Of note, p1@Arx is poorly expressed in neurons of the substantia nigra but strongly expressed in astrocytes of the CB and HIP. Moreover, in CB, the coexpression of p1@Kdm5c and p1@Zfp711 is evident, and the weak expression of p1@Phf8, p2@Phf8, while p1@Arx expression was not detected (Figure 3E; Supplementary Table S2). Collectively, the TSS data obtained both in human and mouse samples strongly support the hypothesis that $K D M 5 C / K d m 5 c, A R X / A r x$, ZNF711/Zfp711, and PHF8/Phf8 constitute a core regulatory network activated consistently in the same or distinctive cell type or brain areas in mammals. 

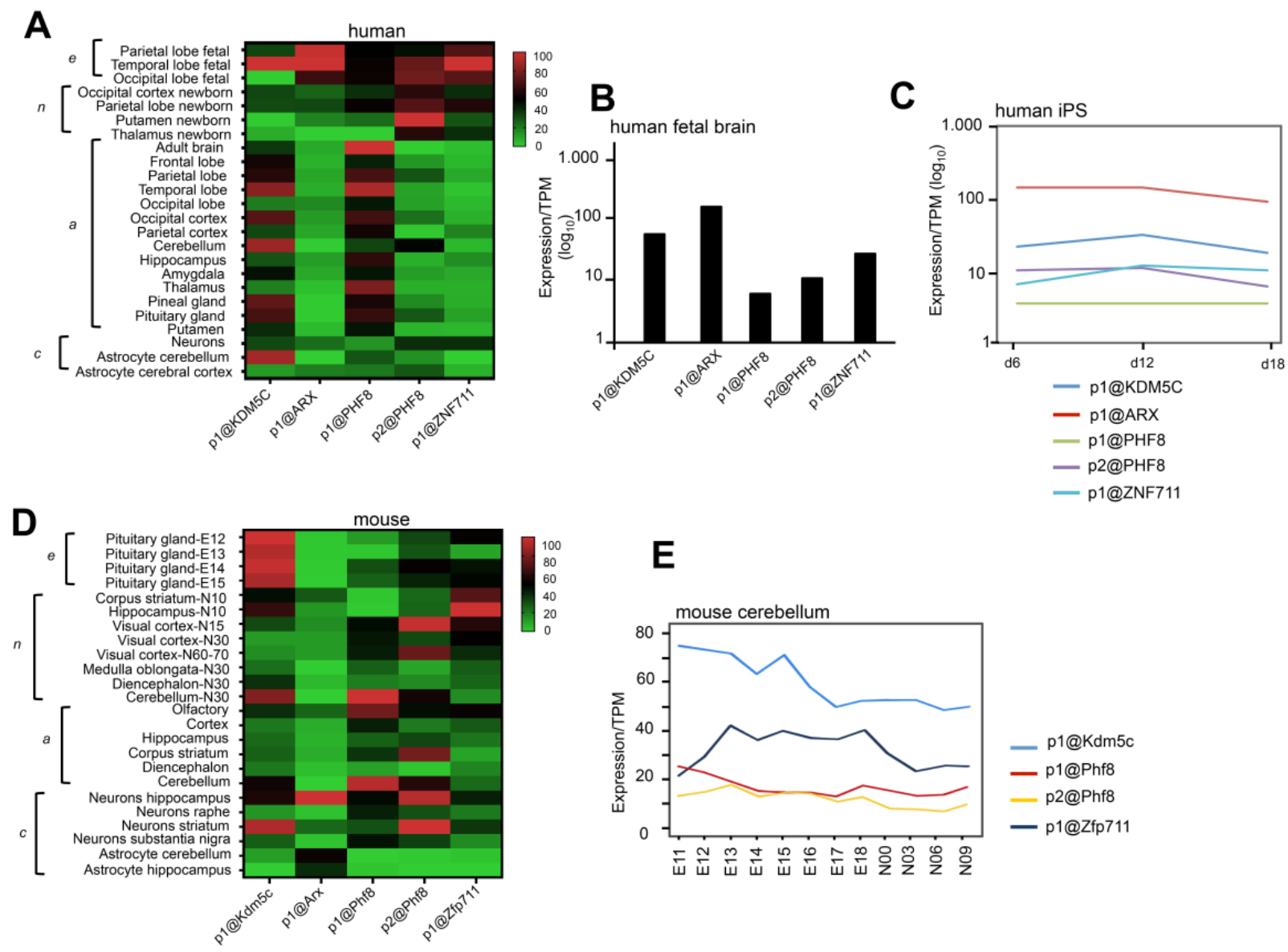

Figure 3. Expression profiles of TSSs in human $(\mathbf{A}-\mathbf{C})$ and mouse $(\mathbf{D}, \mathbf{E})$ brain samples. Heat map showing the TPM expression of all promoters in sub-regions of brains and brain-related primary cells in human (A). Expression profiles of p1@KDM5C, p1@ARX, p1@PHF8, p2@PHF8, and p1@ZNF711 in human fetal brain (B) and in human iPS differentiated to neuron $(C)$, (day 6 = neuro-ectodermal cells; day 12 = neural stem cells; day 18 = early neuronal progenitors). The expression of each CAGE promoter is reported as TPM on the $y$-axis. Heatmap showing the TPM expression of all promoters in sub-regions of brains and brain-related primary cells in mice (D). Expression profiles of p1@Kdm5c, p1@Arx, p1@Phf8, p2@Phf8, and p1@Zfp711 in mouse cerebellum (E). Heatmap's data are normalized and are reported as percentages with respect to the largest value in each data set. Data are available at https:/ / fantom.gsc.riken.jp (accessed on 1 May 2021).

\subsection{KDM5C, ARX, PHF8, and ZNF711 Are Syntenic Genes of Human/Mouse X-Chromosome}

Gene order is not random with regard to gene expression in mammals but is maintained by natural selection because it has a functional significance [41]. We analyzed genome annotations of KDM5C and its regulatory genes on human (UCSC Genome GRCh37/hg19) and mouse (UCSC Genome GRCm38/mm10) genome assembly. As shown in Figure 4, the four genes KDM5C, ARX, PHF8, and ZNF711 are all located in syntenic blocks of X chromosome. Specifically, the nearby genes KDM5C (hg19 chrX: 53,220,50353,254,604) and PHF8 (hg19 chrX: 53,963,113-54,071,569), at physical distance between them of $708 \mathrm{~Kb}-$ map to the region of human $X$ chromosome $p 11.22$. This region is syntenic to the inverted region of the mouse $\mathrm{X}$ chromosome $\mathrm{qF} 3$ where their murine counterparts, $\mathrm{Kdm} 5 \mathrm{c}$ (mm10 chrX: 152,233,229-152,274,535) and Phf8 (mm10 chrX: 151,520,672-151,625,833), map-physical distance between them is $607 \mathrm{Kbs}$. The human ARX (hg19 chrX: 25,021, $811-25,034,082)$ maps to the $\mathrm{Xp} 21.3$ region syntenic to the inverted region of mouse $\mathrm{X}$ chromosome qC3 where maps the murine Arx orthologue (mm10 chrX: 93,286,496-93,298,357) maps; and finally, the human ZNF711 (hg19 chrX: 84,498,997-84,528,368) maps to the Xq21.1 region syntenic to the mouse $X$ chromosome $\mathrm{qE} 1$, where the murine counterpart named Zfp711 maps (mm10 chrX: 112,600,526-112,635,062). Although it lacks an understanding of 
how these clusters may exist in mammals and what their functional significance is, we may assume that - due to their functional relationship during the brain development-KDM5C and its transcriptional regulators may tend to be clustered within the same chromosome.
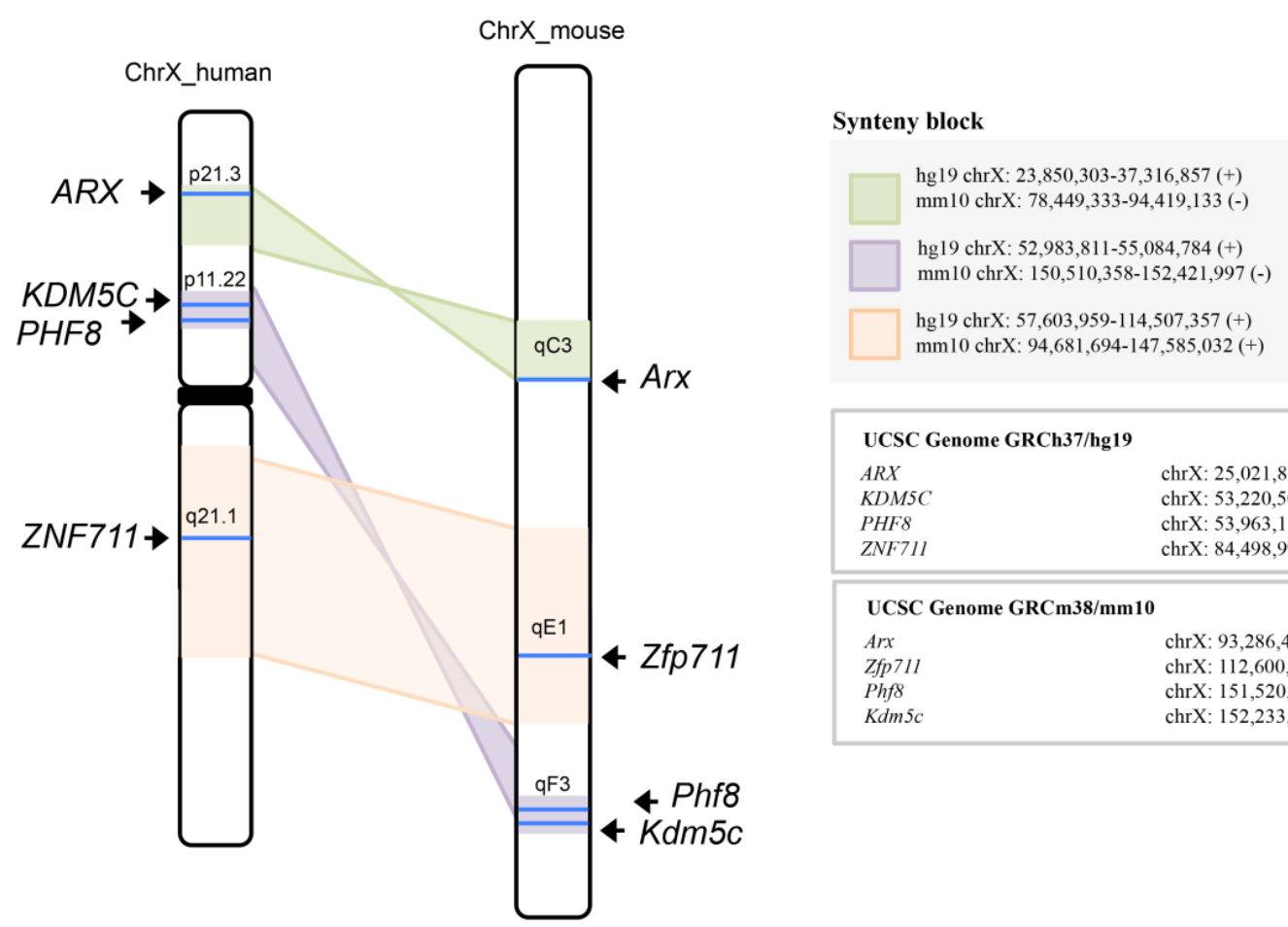

\begin{tabular}{|ll|}
\hline UCSC Genome GRCh37/hg19 & \\
ARX & chrX: $25,021,811-25,034,082$ \\
KDM5C & chrX: $53,220,503-53,254,604$ \\
PHF8 & chrX: $53,963,113-54,071,569$ \\
ZNF711 & chrX: $84,498,997-84,528,368$ \\
\hline \hline & \\
\hline UCSC Genome GRCm38/mm10 & \\
Arx & chrX: $93,286,496-93,298,357$ \\
Zfp711 & chrX: $112,600,526-112,635,062$ \\
Phf8 & chrX: $151,520,672-151,625,833$ \\
Kdm5c & chrX: $152,233,229-152,274,535$ \\
\hline
\end{tabular}

Figure 4. KDM5C, ARX, PHF8, and ZNF711 are located in syntenic segments of human and mouse X chromosome (ChrX_ human and ChrX_mouse, respectively). SynBrowser map of the human and mouse $X$ chromosome and the regions containing the genes (not to scale; http:/ / bioinfo.konkuk.ac.kr/synteny_portal/, accessed on 1 May 2021). Block linkages in the same orientation are labelled in pink (ChrX human Xq21.1 vs. ChrX mouse XqE1), while those in inverted orientation are labelled in green (ChrX human Xp21.3 vs. ChrX mouse XqC3) or in violet (ChrX human Xp11.22 vs. ChrX mouse XqF3). Nucleotide positions of each block are shown.

\subsection{ARX Interacts with PHF8}

In a previous study, we showed that PHF8 and ARX acted cooperatively to induce KDM5C stimulation [5]. Therefore, we wondered whether this activity is mediated by a molecular interaction of PHF8 with ARX. To this end, we evaluated by coimmunoprecipitation (Co-IP) the ability of these regulatory transcriptional factors to interact directly in both endogenous and transiently transfected conditions. As shown in Figure 5A, endogenous ARX was immunoprecipitated by the anti-PHF8 antibody and vice versa, suggesting a direct protein-protein interaction. In addition, the formation of the ARX/PHF8 complex was observed in the coexpression of the HA- and Myc-tagged versions of PHF8 and ARX (Figure 5B). The coexpression of the HA- and Myc-tagged version of PHF8 and ZNF711 was tested as the positive control of their direct interaction, as previously described [34] (Figure 5B). Thus, our results have revealed a direct connection between PHF8 and ARX, expanding the regulatory intersection of KDM5C stimulation (Figure 5C). 


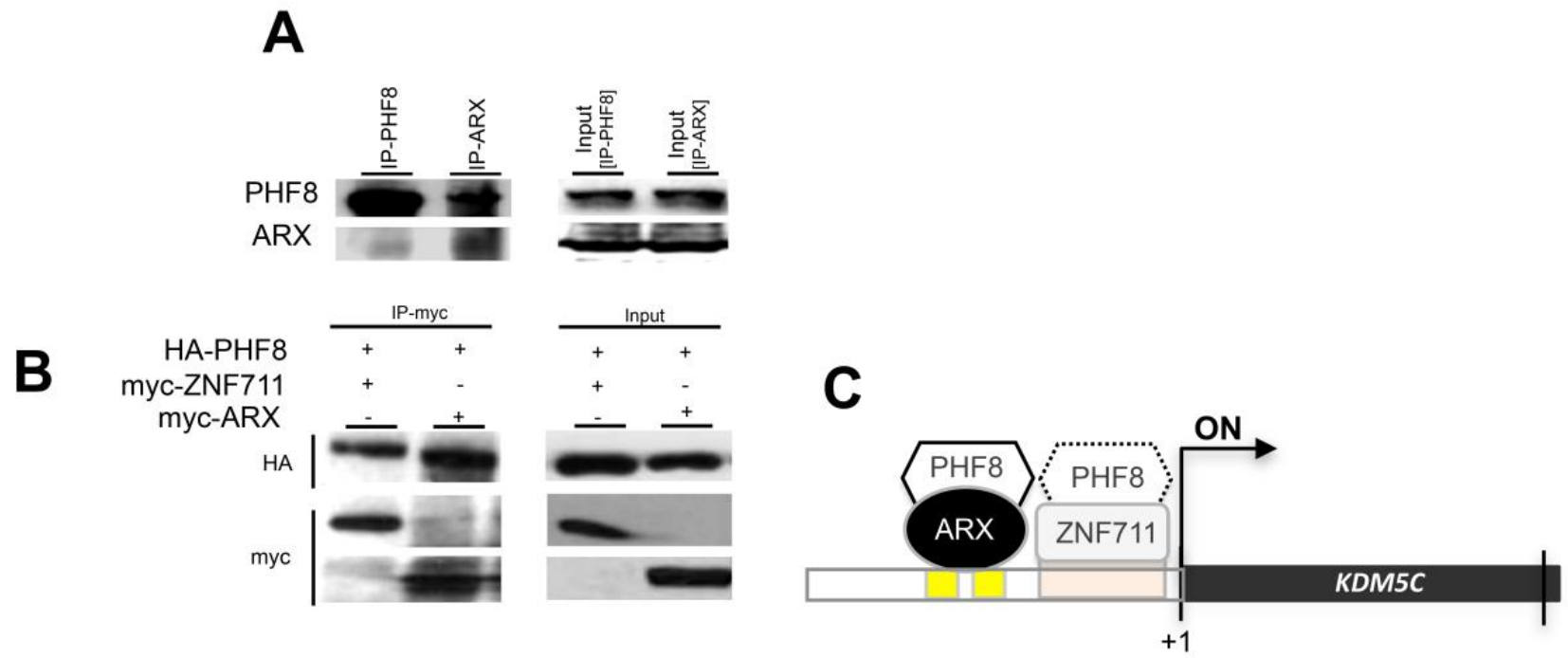

Figure 5. Co-immunoprecipitation of PHF8 and ARX in SH-SY5Y cells. Co-IP of endogenous PHF8 and ARX and vice versa. Cell lysates were subjected to immunoprecipitation with anti-PHF8 or anti-ARX antibodies. The presence of PHF8 and ARX in the cell extracts prior to immunoprecipitation was checked using anti-PHF8 and anti-ARX antibodies (Input) (A). Cells were transfected with a pCMV vector to express HA tagged-PHF8 (120 kDa), or Myc tagged-ARX (75 kDa), or Myc tagged-ZNF711 (100 kDa) (B). The cell lysates were subjected to immunoprecipitation with anti-HA or anti-Myc- antibodies. The presence of PHF8-HA, ZNF711-Myc, and ARX-Myc in cell extracts prior to immunoprecipitation was checked using anti-HA and anti-Myc antibodies (Input). Regulatory interactions with activating effects on KDM5C transcription are shown in the carton $(\mathbf{C})$.

\subsection{Analysis of KDM5C and H3K4me3 Levels in NDD Patient-Derived Lymphoblastoid Cell Lines}

We set out to investigate on KDM5C expression and H3K4me3 levels in Epstein-Barr virus (EBV)-transformed lymphoblastoid cell lines (LCLs) obtained from eight NDD patients (P) with mutations in KDM5C or in its three regulators (Table 1). Specifically, the NDD P1 and P2 LCL cells were obtained from two unrelated male patients, presenting mild cognition defects, who carry the same in-frame ARX duplication c.429_452dup24 [p.A148_A155dup] (Figure 6A; [4,18,42,43]). From P3 to P6, LCLs were obtained from four male patients with mutations in KDM5C characterized by a variable degree of ID and comorbidities. Specifically, the nonsense mutations c.3864G $>$ A [p.W1288X] [10,44] and c.1599delC [p.W534Gfs*15] [45] from patients P3 and P4, respectively, are both characterized by severe ID with epilepsy (Table 1); the missense mutation c.1162G $>C$ [p.A388P] [10] in patient P5, presents ID with mild facial dysmorphism (Table 1); and the missense mutation c.2248C > T [p.R750W] [46] in patient P6, presents severe ID and speech impairment (Table 1). P7 LCLs were obtained by a male patient with ID and cleft/lip palate who carries the nonsense mutation c.1050_1061del12 [p.P314fs*] in PHF8 [23] (Table 1). Finally, P8 LCLs were obtained from a male patient with mild ID who carries the missense mutation c.731T>C [p.I244T] in ZNF711 [29] (Table 1). 
Table 1. List of disease mutations analyzed in this study.

\begin{tabular}{|c|c|c|c|c|c|}
\hline Gene & cDNA Change & Protein Change & Mutation & Clinical Features & Reference \\
\hline \multirow[t]{2}{*}{$A R X$} & c.298_330dup33 & p.Ala105_115dup & duplication & DEE1, severe ID & {$[4,18,42,43]$} \\
\hline & c.429_452dup24 & p.Ala148_155dup & duplication & mild ID & {$[4,18,42,43]$} \\
\hline \multirow[t]{4}{*}{ KDM5C } & c. $1162 \mathrm{G}>\mathrm{C}$ & p.Ala388Pro & missense & ID, mild dysmorphism & [10] \\
\hline & c.3864G >A & p.Trp1288X & nonsense & $\begin{array}{l}\text { severe ID, spasticity, epilepsy, } \\
\text { microcephaly }\end{array}$ & {$[10,44]$} \\
\hline & c.1599delC & p.Trp534Glyfs*15 & nonsense & severe ID, epilepsy, spasticity & [45] \\
\hline & c. $2248 \mathrm{C}>\mathrm{T}$ & p.Arg750Trp & missense & severe ID, speech impairment & [46] \\
\hline PHF8 & c.1050_1061del12 & p.Pro314fs* & nonsense & $\begin{array}{l}\text { mild to borderline ID, cleft lip and } \\
\text { cleft palate }\end{array}$ & [23] \\
\hline ZNF711 & c. $731 \mathrm{~T}>\mathrm{C}$ & p.Ile244Thr & missense & mild to borderline ID, speech delay & [29] \\
\hline
\end{tabular}

ID, Intellectual disability; DEE1, Developmental epileptic encephalopathy-type 1.

By real-time PCR, we firstly detected a variable expression of the KDM5C mRNA in patient-derived LCLs with respect to the control male-derived LCLs (Figure 6A): in the $A R X, \mathrm{P} 1$ and $\mathrm{P} 2$ patients expressed $57 \%$ and $56 \%$ lower compared with the WT controls, respectively; in KDM5C, P3-P6 expressed $41 \%, 47 \%, 60 \%$, and 29\%, respectively; in PHF8, P7 expressed 31\%; and in ZNF711, P8 expressed 5\%. Secondly, by Western blotting we tested the protein levels of KDM5C and of its main substrate trimethylation of lysine 4 of histone $\mathrm{H} 3$ (H3K4me3). In line with the real-time-PCR data, we found that all mutations in $A R X, P H F 8$, and ZNF711 lead to a decrease in KDM5C protein content (Figure 6B). This defect inversely correlates with an increase in the H3K4me3 level, potentially as the result of the compromised demethylase activity of KDM5C (Figure 6B). These findings suggest that the mutations in $K D M 5 C$ regulators act on the KDM5C-H3K4m3 pathway as partial loss-of-function because of a variable basal activity depending on the type of mutations. About KDM5C mutations, the nonsense KDM5C p.W1288X and KDM5C p.W534Gfs*15 mutations operate as loss-of-function mutations since they are incapable of synthesizing the relative proteins, and thus, of demethylase H3K4me3 (Figure 6B). Noteworthily, the missense mutation KDM5C p.A388P, showed a protein intensity band of KDM5C and H3K4me3 similar to that of the WT. In line with the predicted impact on the protein conformation reported previously [47], we conclude that this missense mutation does not impair the demethylase activity but instead alters the binding to other proteins, perhaps involved into the recruitment of KDM5C to specific target promoters.

On the contrary, for the missense mutation p.R750W, the respective KDM5C protein band was severely reduced and the H3K4me3 band increased, with respect to the control. For this alteration, one explanation is that the substitution of the evolutionarily conserved arginine with a tryptophan residue in C5HC2-Zinc Finger domain of KDM5C [46] might cause a reduced protein stability followed by a protein degradation process operating as partial loss-of-function. We conclude that in NDD patient-derived cell lines, as a result of the KDM5C decrease (partial LoF) or the KDM5C absence (LoF), the global levels of H3K4me3 signal increase determine a perturbation of chromatin remodeling. 

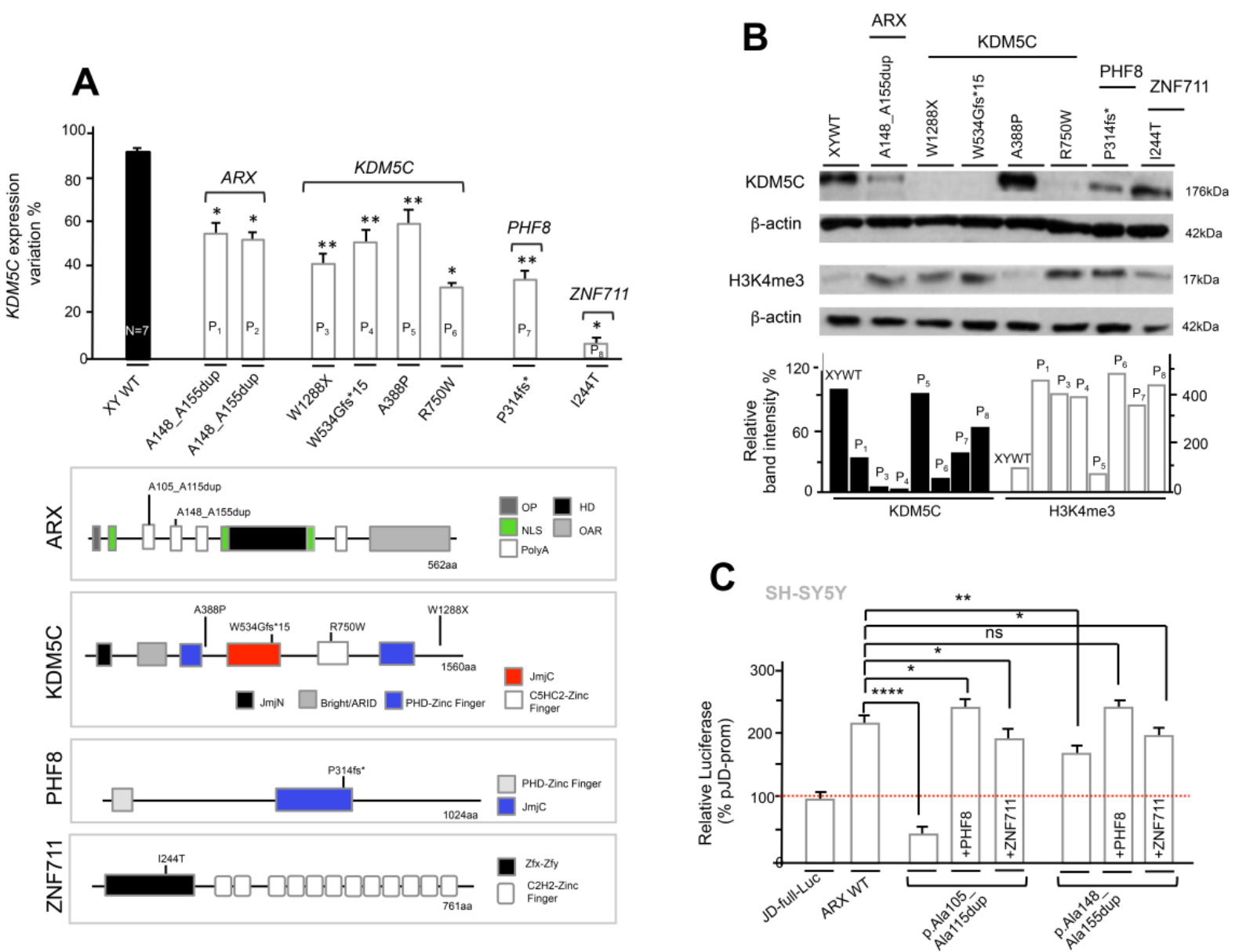

Figure 6. KDM5C defects associated to NDD mutations. KDM5C expression in lymphoblastoid cell lines obtained from distinct NDD patients mutated in ARX, KDM5C, PHF8, and ZNF711. The percentages indicate the expression variation with respect to the WT, which has a mean of seven $(n=7)$ different cell lines from male age-matched males. The transcript analysis was performed in triplicate and the samples were normalized to $18 S$. The bars indicate the mean \pm standard error of three independent experiments (top panel). Positions of the studied mutations are shown in the cartoon (bottom panel). OP, Octapeptide; polyA, polyalanine tracts; NLS, Nuclear localization sequences; HD, homeodomain; OAR, Aristaless domain; JmN, jumonji-N domain; ARID, AT-rich interacting domain; PHD, plant homeodomain box domain; JmjC, jumonji-C catalytic domain; ZF, zinc finger domain; PLU-1, PLU-1-like domain; Zfx/Zfy, Transcription activation domain (A). Western blotting for KDM5C and its substrate H3K4me3 was performed on cellular extracts from LCLs. The $\beta$-actin antibody ( $\beta$-actin) was used as a loading control. Each experiment was repeated with two independent LCL protein extracts with similar results (B). Effects of PHF8 and ZNF711 overexpression on KDM5C 5' UTR region in cotransfection with ARX polyalanine elongated mutants. Each luciferase assay was performed in duplicate in four independent experiments. The bars indicate the mean \pm standard error of four independent experiments $(\mathbf{C}) .{ }^{*} p<0.05 ;{ }^{* *} p<0.005 ;{ }^{* * * *} p<0.0005$; ns, not significant.

\subsection{The Defective ARX-Dependent Transactivation of KDM5C Is Balanced by PHF8 or ZNF711 Overexpression}

We previously showed that the loss of promoter stimulation of KDM5C caused by PHF8 mutant proteins was rescued by ZNF711 coexpression; while the loss of KDM5C activity of mutant ZNF711 proteins was not compensated by WT PHF8 coexpression [5]. Expanding this analysis, we cotransfected the JD-full-Luc construct with full-length Myc-tagged ARX (GenBank: NM_139058.2) WT and p.Ala105_Ala115dup and p.Ala148_Ala155dup mutant expressing vectors into SH-SY5Y cells (Figure 6C; Table 1). We observed that the coexpression of ZNF711 with PHF8 or individually led to normalization of the interfering defects of ARX-KDM5C interaction, associated with p.Ala105_Ala115dup and p.Ala148_Ala155dup mutations, increasing the luciferase quantity score back to the level 
obtained with the ARX WT construct (Figure 6C). This suggests that both the overexpression of ZNF711 and PHF8 might balance the impairment of the stimulation of KDM5C promoter in case of $A R X$ mutations with partial loss-of-function.

\section{Discussion}

Spatiotemporal gene expression differences across specific brain regions are guided by regulatory interactions through epigenetic modifications and transcriptional changes. Here, we reveal that a set of NDD genes, encoding the demethylases KDM5C and PHF8 and the transcription factors ARX and PHF8, is highly interconnected, showing a strongly coordinated expression pattern across neurogenesis and during neuronal differentiation of hiPSCs. These genes show a peculiar coexpression pattern in hippocampal and neocortical regions, with two intersected cardinal areas involved in flexible cognition and social behavior [48]. Moreover, three of them-KDM5C, ARX, and PHF8-show high expression levels at the timing of neuronal proliferation with a decrease during neuronal migration and synaptogenesis-two highly vulnerable processes underpinning neural circuit development [2]. By contrast, ZNF711 shows an oscillatory trend with a very low expression level during the proliferation phase that increases, reaching a peak at the beginning of neuronal migration and synaptogenesis (Figure 7A). In embryonic and neonatal mouse brain, transcript and protein levels of $K d m 5 c$, Arx, Phf8, and Zfp711 show a similar framework: all four genes, and their relative proteins, are mainly coexpressed during the early stages of neurogenesis and neuronal migration with a gradual decrease until to the birth (Figure 7A).

A Human-Multiple Cortical Areas - SMART-seq

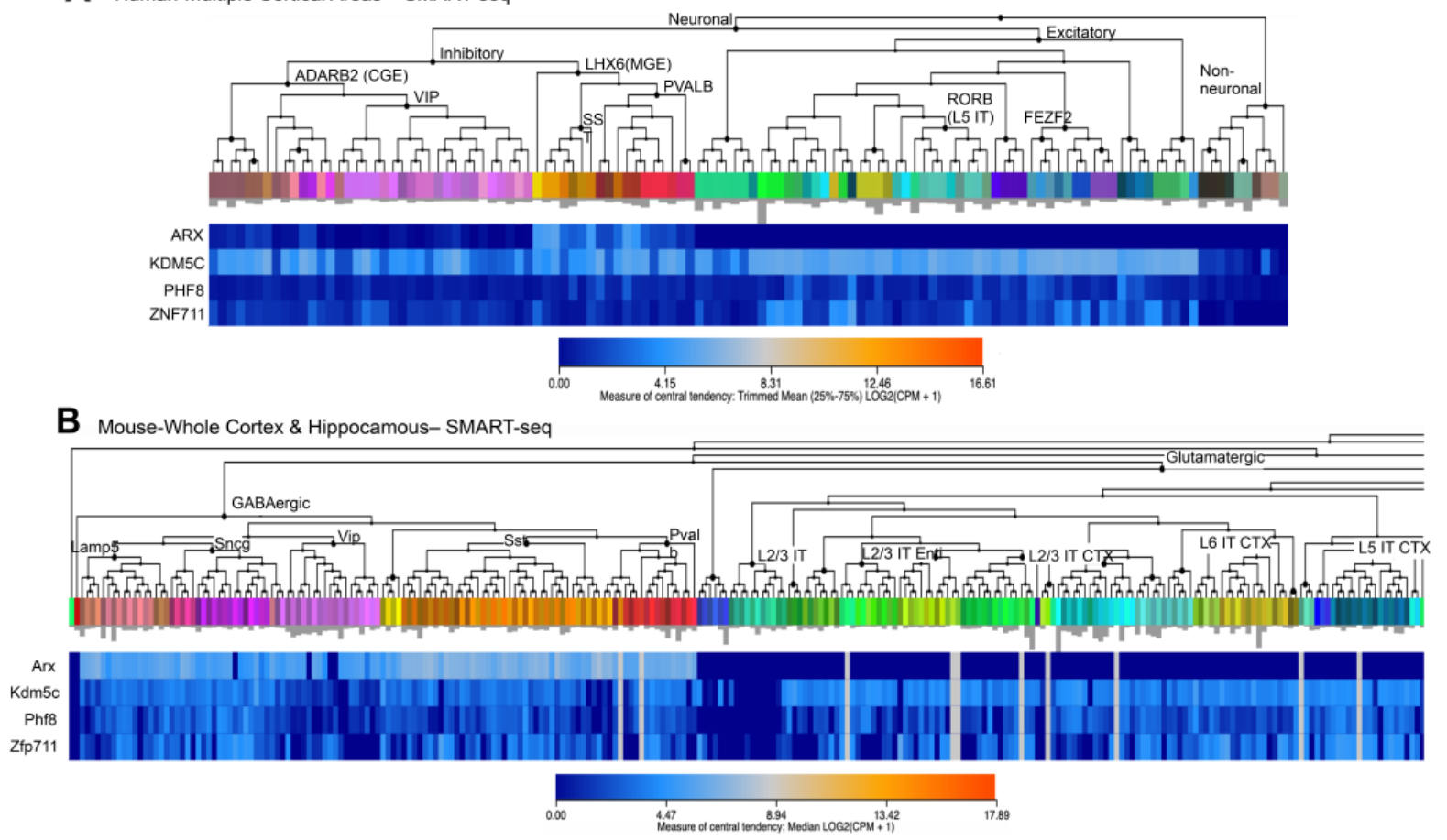

Figure 7. Heatmaps showing the expression profiles of $K D M 5 C / K d m 5 c$ and its regulatory genes $A R X / A r x, P H F 8 / P h f 8$, and ZNF711/Zfp711. Data were obtained from Allen Brain Atlas/Transcriptomics Explorer in human (A) and mouse (B). Original images can be accessed freely at https: / / celltypes.brain-map.org/rnaseq/ (accessed on 10 July 2021). 
Additional data based on FANTOM5 CAGE database reinforce the concept that this set of NDDs shows overlapping time-specific transcriptional modules. They operate during brain development-where they are involved in neuronal cell specification-and in adult brain areas-where they control neuronal plasticity and functioning. Noteworthily, CAGE analysis was successfully applied to characterize regulatory intersection among genes found mutated in other NDDs-such as Rett Syndrome-and build the atlas of TSS across major human cell-types and tissues [32,49]. The entry into the scene of the three regulating genes at various times of neurogenesis suggests that transcription of the epigenetic eraser KDM5C has a multi-level regulatory governance-particularly in human-capable of ensuring the correct dosage of KDM5C in response to specific stimuli at the proper time and in the proper tissue area or cell. Moreover, further investigation on the roles of these transcriptional modules in specific cell subtypes, such as glutamatergic neurons, GABAergic neurons and glia cells should be performed. To this end, single-cell RNA sequencing studies allow defining single cell coexpression landscapes and identifying targeted cells for potential treatments [50]. Remarkably, as reported in the Human Protein Atlas dataset (https:/ / www.proteinatlas.org/ accessed on 10 July 2021), KDM5C and its three regulatory proteins ARX, ZNF711 and PHF8 are all expressed in the corpus callosum, in human, pig, and mouse (Table S4). Deciphering whether these subsets of NDD genes are mutually related to the development of callosal projection neurons (CPN), will shed light on the molecular mechanisms controlling the formation of the corpus callosum, a structure that connect the two cortical hemispheres. Noteworthily, one of the allelic disorders caused by ARX mutations is Proud syndrome, presenting as a main clinical features the agenesis of the corpus callosum [51]. Nevertheless, no gross alterations in the corpus callosum have been reported in patients mutated in KDM5C, ZNF711, and PHF8 [10,23,29,44-46].

Since defects in GABAergic neurons feature prominently in NDDs, another interesting aspect that requires deep investigation is that whether this subset of NDD genes is mutually involved in differentiation and functioning of GABAergic interneurons. By interrogating the Allen Brain Atlas RNAseq database, it is evident that they are mutually coexpressed in specific subsets of GABAergic neurons, both in human and mouse (Figure 7A,B; [52]). The direct involvement of ARX and KDM5C in GABAergic-related activities has been already reported $[4,5,9,53]$; while it is unknown the role of ZNF711 and PHF8 is unknown. Furthermore, 3D human organoids from patients could help to identify in detail the functional impact of each of them on the production of GABAergic neurons and whether they could alter the balance between GABAergic and glutamatergic neurons.

Another interesting observation is that the localization of $K D M 5 C$ and its regulatory genes is conserved within synthenic blocks of $X$ chromosomes in human and mouse. This conserved localization parallels their coexpression profiles in human and mouse nervous system, suggesting that the coordinated waves of gene expression during neurogenesis and in specific brain areas is controlled by conserved regulatory mechanisms. Indeed, binding sites for key lineage and/or tissue-specific transcription factors could be simultaneously recruited at the enhancers and active promoters of this set of X-linked genes. On the other hand, clustering of genes expressed during the early phases of brain development could be advantageous to assemble chromatin neighbourhoods with open conformation. Moreover, further studies are required to define whether they fall into the same topologically associating domain (TAD) and/or share common regulatory players.

Chromatin perturbations associated to mutations in neurodevelopmental genes damage vulnerable brain processes such as cognition development and neuronal homeostasis [2,3]. Notably, in patient-derived LCLs with mutations in KDM5C, ARX, PHF8, and ZNF711, we detected aberrant dosage of KDM5C and H3K4me3.

This suggests that the neurological diseases linked to this set of genes can be considered as X-linked chromatinopathies. Specifically, the aberrant levels of KDM5C and H3K4me3 in ID males mutated in KDM5C, ZNF711, PHF8, and ARX highlight a variable chromatin defect as a consequences of the type of disease mutation. This secondary disease hit could cause a relaxation in the chromatin conformation and compromise nervous system 
development. In reference to this, we observed a direct relationship between the severity of the KDM5C-H3K4me3 defect with the severity of cognition impairment. This feature highlights the value of KDM5C as a disease epigenetic marker to predict ID severity caused by mutations in its gene (direct effect) or in its regulator genes (indirect effect) (Figure 8B). Obviously, the KDM5C-H3K4m3 axis is not the solely damaged process, but other pathways can be altered as a result of mutations in genes involved in pleiotropic activities, such as ARX, ZNF711, or PHF8. We therefore propose a model explaining that the varying severity of ID, a common clinical core associated to the four disease genes, could be explained through the impact on KDM5C-H3K4me3 axis (Figure 8B). On the other hand, the identification of further regulatory elements of the KDM5C transcriptional axis (e.g., cofactors, interactors, non-coding RNAs, etc.) and molecular compounds capable of modulating its expression levels (e.g., epidrugs, small peptides, etc.) will contribute to deeply define how to counteract a KDM5C deficit in time-regulated windows of cognition development. In this direction are the recent findings on the functional interactions of KDM5C with lysine methyltransferase 2A (KMT2A; MIM 159555), responsible for Wiedemann-Steiner Syndrome (WDSTS; MIM:605130), proving that this eraser-writer pair acts in a mutually suppressive mode, ameliorating their associated disorders [9]. More interestingly, we have shown that in vitro and in vivo treatments with suberanilohydroxamic acid (SAHA) is a potent reversible pan-histone deacetylase (HDAC) inhibitor, is able to correct the KDM5C-H3K4me3 pathway [5].

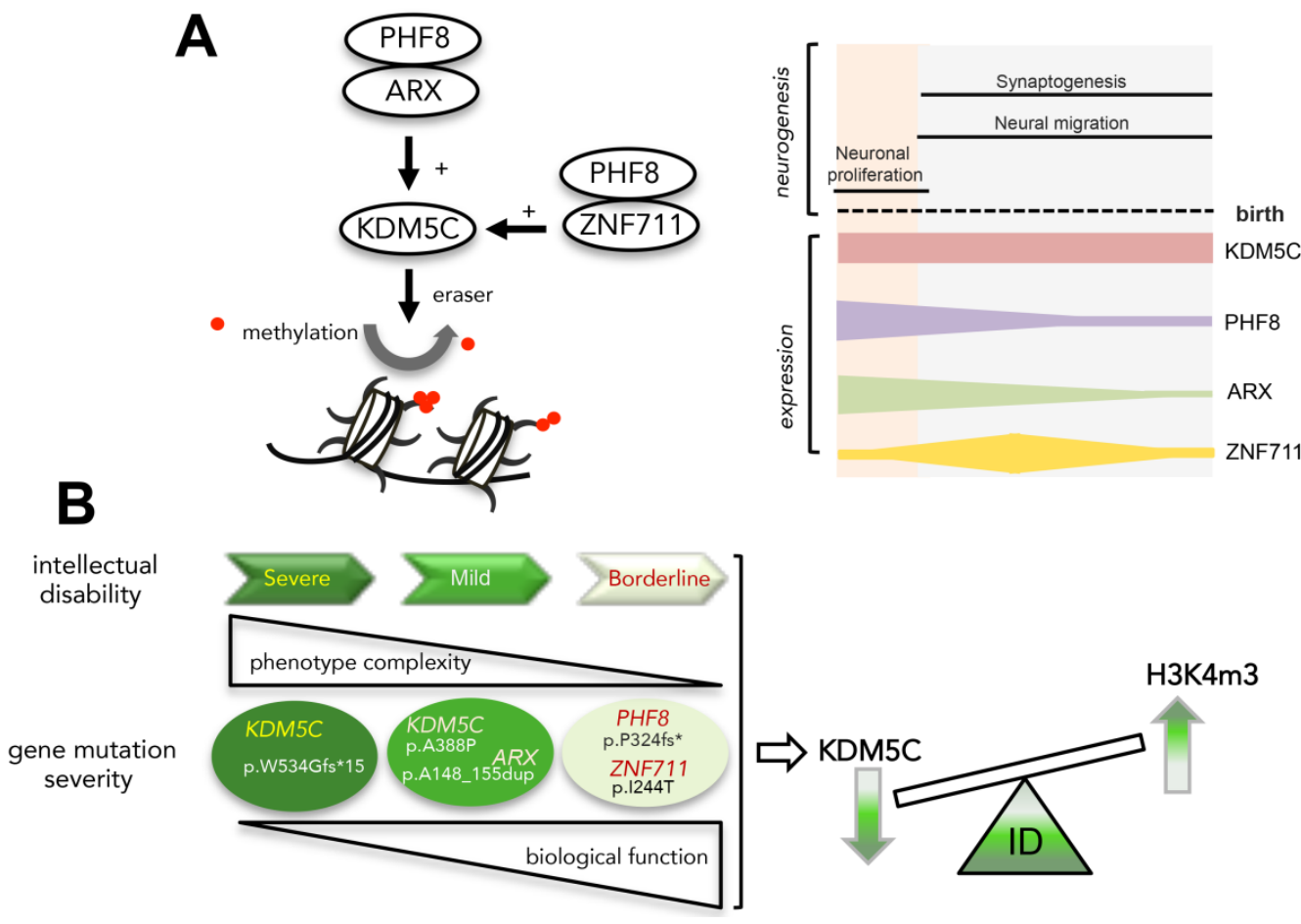

Figure 8. KDM5C is an epigenetic marker of ID severity. Regulatory governance of KDM5C transcription (A, left panel); and expression profiles of $K D M 5 C$ and its regulatory genes during human neurogenesis (A, right panel); functional correlation between mutation severity and ID (B).

A peculiar aspect of this highly connected regulatory axis is the capacity to rebalance the partial loss activity of expanded polyalanine tracts in ARX via overexpression of the coregulatory proteins ZNF711 or PHF8. Along the same line, we previously found that ZNF711 overexpression is also capable of balancing the partial loss activity of PHF8 mutant proteins [5]. Collectively, these findings suggest that PHF8 and ZNF711 may act as gene modifiers of ARX-disease phenotypes; thus, variants in these NDD genes may contribute to the phenotype variability from West syndrome to Partington syndrome to mild ID caused by ARX polyalanine expansions. Even though more studies are needed to 
fully understand the peculiar activities of this intersected NDD pathway, we propose that the $K D M 5 C$ transcription has a multi-level regulatory governance coupled to a positive feedback mechanism. In addition, because the expression profiles of KDM5C regulatory genes are mutually related to multiple cellular subtypes, as callosal projection neuron and interneurons, they could be involved in differentiation and functioning of varying neuronal cell types.

\section{Conclusions}

Our findings indicate that KDM5C is an epigenetic marker of developmental signatures through which we may estimate the functional impact of disease mutations on cognition processes and that it represents a convergent point for therapeutic intervention for a set of X-linked chromatinopathies.

Supplementary Materials: The following are available online at https:/ / www.mdpi.com/article/ 10.3390/genes12071088/s1, Figure S1: Gene expression levels of KDM5C and its regulatory genes throughout neuronal differentiation. Figure S2: Expression analysis of $K d m 5 c, A r x$, Phf8 and Zfp711 in adult male mouse brain (P56). Figure S3: Zenbu genome browser views of gene locus for human KDM5C, ARX, PHF8, and ZNF711. Table S1: CAGE annotation from Zenbu database. Table S2: Human and murine expression data of CAGE obtained from Zenbu database. Table S3: Oligonucleotides used for transcript analysis. Table S4: RNA expression summary of KDM5C and its regulatory genes in the corpus callosum (from The Human Protein Atlas datasets; https: //www.proteinatlas.org/, accessed on 1 May 2021).

Author Contributions: L.P. conceived the study, carried out in silico, in vitro, and in vivo studies; A.P. performed co-IP and LCL analysis; M.B.L. and H.v.B. contributed to the implementation of the research and the interpretation of results; M.G.M. coordinated the study and drafted the manuscript with the input from L.P. All authors have read and agreed to the published version of the manuscript.

Funding: This research was funded by Jerome Jejune Foundation grants (1372-MM2015A), Telethon Foundation grant (GGP14198) and Italian Ministry of Economic Development grant (F/050011/02/X32) to M.G.M.

Institutional Review Board Statement: Ethical approval for studies in animals was obtained by the Italian Ministry of Health (DLgs116/92) in accordance with the Institutional Animal Care guidelines of the Institute of Genetics and Biophysics Adriano Buzzati-Traverso.

Informed Consent Statement: The study was approved by the Medical Ethical Committee ArnhemNijmegen, the Netherlands and adhered to the Declaration of Helsinki. Written informed consent was obtained from each individual.

Data Availability Statement: Publicly archived datasets analyzed: FANTOM5 database https:// fantom.gsc.riken.jp, accessed on 18 June 2021; Human Brain Transcriptome Atlas https:/ /hbatlas.org/ hbtd/basicSearch.pl, accessed on 1 May 2021; Liber Institute for Brain development http://stemcell. libd.org/scb/, accessed on 1 May 2021; Allen Mouse Brain Atlas https://mouse.brain-map.org/, accessed on 1 May 2021; UCSC https:/ / genome.ucsc.edu/; Cell Types Database https:/ / celltypes. brain-map.org/rnaseq/, accessed on 1 May 2021.

Acknowledgments: We thank IGB Mouse Facility and all members of Miano laboratory for useful discussions.

Conflicts of Interest: The authors declare that they have no conflict of interest.

\section{References}

1. Meaney, M.J.; Ferguson-Smith, A. Epigenetic regulation of the neural transcriptome: The meaning of the marks. Nat. Neurosci. 2010, 13, 1313-1318. [CrossRef] [PubMed]

2. Mossink, B.; Negwer, M.; Schubert, D.; Kasri, N.N. The emerging role of chromatin remodelers in neurodevelopmental disorders: A developmental perspective. Cell. Mol. Life Sci. 2021, 78, 2517-2563. [CrossRef] [PubMed]

3. Ciptasari, U.; Van Bokhoven, H. The phenomenal epigenome in neurodevelopmental disorders. Hum. Mol. Genet. 2020, 29, R42-R50. [CrossRef] 
4. Poeta, L.; Fusco, F.; Drongitis, D.; Shoubridge, C.; Manganelli, G.; Filosa, S.; Paciolla, M.; Courtney, M.; Collombat, P.; Lioi, M.B.; et al. A Regulatory Path Associated with X-Linked Intellectual Disability and Epilepsy Links KDM5C to the Polyalanine Expansions in ARX. Am. J. Hum. Genet. 2013, 92, 114-125. [CrossRef]

5. Poeta, L.; Padula, A.; Attianese, B.; Valentino, M.; Verrillo, L.; Filosa, S.; Shoubridge, C.; Barra, A.; Schwartz, C.E.; Christensen, J.; et al. Histone demethylase KDM5C is a SAHA-sensitive central hub at the crossroads of transcriptional axes involved in multiple neurodevelopmental disorders. Hum. Mol. Genet. 2019, 28, 4089-4102. [CrossRef]

6. Shen, E.; Shulha, H.P.; Weng, Z.; Akbarian, S. Regulation of histone H3K4 methylation in brain development and disease. Philos. Trans. R. Soc. B Biol. Sci. 2014, 369, 20130514. [CrossRef]

7. Iwase, S.; Lan, F.; Bayliss, P.; De La Torre-Ubieta, L.; Huarte, M.; Qi, H.; Whetstine, J.R.; Bonni, A.; Roberts, T.M.; Shi, Y. The X-Linked Mental Retardation Gene SMCX/JARID1C Defines a Family of Histone H3 Lysine 4 Demethylases. Cell 2007, 128, 1077-1088. [CrossRef]

8. Scandaglia, M.; Lopez-Atalaya, J.P.; Medrano-Fernandez, A.; Lopez-Cascales, M.T.; del Blanco, B.; Lipinski, M.; Benito, E.; Olivares, R.; Iwase, S.; Shi, Y.; et al. Loss of Kdm5c Causes Spurious Transcription and Prevents the Fine-Tuning of Activity-Regulated Enhancers in Neurons. Cell Rep. 2017, 21, 47-59. [CrossRef] [PubMed]

9. Vallianatos, C.N.; Raines, B.; Porter, R.S.; Bonefas, K.M.; Wu, M.C.; Garay, P.M.; Collette, K.M.; Seo, Y.A.; Dou, Y.; Keegan, C.E.; et al. Mutually suppressive roles of KMT2A and KDM5C in behaviour, neuronal structure, and histone H3K4 methylation. Commun. Biol. 2020, 3, 278. [CrossRef]

10. Jensen, L.R.; Amende, M.; Gurok, U.; Moser, B.; Gimmel, V.; Tzschach, A.; Janecke, A.; Tariverdian, G.; Chelly, J.; Fryns, J.-P.; et al. Mutations in the JARID1C Gene, Which Is Involved in Transcriptional Regulation and Chromatin Remodeling, Cause X-Linked Mental Retardation. Am. J. Hum. Genet. 2005, 76, 227-236. [CrossRef]

11. Abidi, F.E.; Holloway, L.; Moore, C.A.; Weaver, D.D.; Simensen, R.J.; Stevenson, R.E.; Rogers, R.C.; Schwartz, C.E. Mutations in JARID1C are associated with X-linked mental retardation, short stature and hyperreflexia. J. Med. Genet. 2008, 45, 787-793. [CrossRef]

12. Vallianatos, C.N.; Farrehi, C.; Friez, M.J.; Burmeister, M.; Keegan, C.E.; Iwase, S. Altered Gene-Regulatory Function of KDM5C by a Novel Mutation Associated with Autism and Intellectual Disability. Front. Mol. Neurosci. 2018, 11, 104. [CrossRef]

13. Adegbola, A.; Gao, H.; Sommer, S.; Browning, M. A novel mutation inJARID1C/SMCX in a patient with autism spectrum disorder (ASD). Am. J. Med. Genet. Part A 2008, 146A, 505-511. [CrossRef]

14. Brookes, E.; Laurent, B.; Ounap, K.; Carroll, R.; Moeschler, J.B.; Field, M.; Schwartz, C.E.; Gecz, J.; Shi, Y. Mutations in the intellectual disability gene KDM5C reduce protein stability and demethylase activity. Hum. Mol. Genet. 2015, 24, $2861-2872$. [CrossRef] [PubMed]

15. Shoubridge, C.; Fullston, T.; Gecz, J. ARX spectrum disorders: Making inroads into the molecular pathology. Hum. Mutat. 2010, 31, 889-900. [CrossRef]

16. Kato, M.; Das, S.; Petras, K.; Kitamura, K.; Morohashi, K.-I.; Abuelo, D.N.; Barr, M.; Bonneau, D.; Brady, A.F.; Carpenter, N.J.; et al. Mutations ofARX are associated with striking pleiotropy and consistent genotype-phenotype correlation. Hum. Mutat. 2004, 23, 147-159. [CrossRef]

17. Kato, M.; Saitoh, S.; Kamei, A.; Shiraishi, H.; Ueda, Y.; Akasaka, M.; Tohyama, J.; Akasaka, N.; Hayasaka, K. A Longer Polyalanine Expansion Mutation in the ARX Gene Causes Early Infantile Epileptic Encephalopathy with Suppression-Burst Pattern (Ohtahara Syndrome). Am. J. Hum. Genet. 2007, 81, 361-366. [CrossRef] [PubMed]

18. Laperuta, C.; Spizzichino, L.; D’Adamo, P.; Monfregola, J.; Maiorino, A.; D’Eustacchio, A.; Ventruto, V.; Neri, G.; D’Urso, M.; Chiurazzi, P.; et al. MRX87 family with Aristaless Xdup24bp mutation and implication for polyAlanine expansions. BMC Med. Genet. 2007, 8, 25. [CrossRef]

19. Chaste, P.; Nygren, G.; Anckarsäter, H.; Råstam, M.; Coleman, M.; Leboyer, M.; Gillberg, C.; Betancur, C. Mutation screening of theARX gene in patients with autism. Am. J. Med. Genet. Part B: Neuropsychiatr. Genet. 2007, 144B, 228-230. [CrossRef] [PubMed]

20. Poeta, L.; Drongitis, D.; Verrillo, L.; Miano, M.G. DNA Hypermethylation and Unstable Repeat Diseases: A Paradigm of Transcriptional Silencing to Decipher the Basis of Pathogenic Mechanisms. Genes 2020, 11, 684. [CrossRef]

21. Nasrallah, M.P.; Cho, G.; Simonet, J.C.; Putt, M.E.; Kitamura, K.; Golden, J.A. Differential effects of a polyalanine tract expansion in Arx on neural development and gene expression. Hum. Mol. Genet. 2011, 21, 1090-1098. [CrossRef]

22. Mattiske, T.; Lee, K.; Gecz, J.; Friocourt, G.; Shoubridge, C. Embryonic forebrain transcriptome of mice with polyalanine expansion mutations in theARXhomeobox gene. Hum. Mol. Genet. 2016, 25, 5433-5443. [CrossRef] [PubMed]

23. Laumonnier, F.; Holbert, S.; Ronce, N.; Faravelli, F.; Lenzner, S.; Schwartz, C.E.; Lespinasse, J.; Van Esch, H.; Lacombe, D.; Goizet, C.; et al. Mutations in PHF8 are associated with X linked mental retardation and cleft lip/cleft palate. J. Med. Genet. 2005, 42, 780-786. [CrossRef] [PubMed]

24. Abidi, F.E.; Miano, M.G.; Murray, J.C.; Schwartz, C.E. A novel mutation in the PHF8 gene is associated with X-linked mental retardation with cleft lip/cleft palate. Clin. Genet. 2007, 72, 19-22. [CrossRef] [PubMed]

25. Chen, X.; Wang, S.; Zhou, Y.; Han, Y.; Li, S.; Xu, Q.; Xu, L.; Zhu, Z.; Deng, Y.; Yu, L.; et al. Phf8 histone demethylase deficiency causes cognitive impairments through the mTOR pathway. Nat. Commun. 2018, 9, 114. [CrossRef]

26. Ni, W.; Perez, A.A.; Schreiner, S.; Nicolet, C.M.; Farnham, P.J. Characterization of the ZFX family of transcription factors that bind downstream of the start site of CpG island promoters. Nucleic Acids Res. 2020, 48, 5986-6000. [CrossRef] 
27. Tarpey, P.S.; Smith, R.; Pleasance, E.; Whibley, A.; Edkins, S.; Hardy, C.; O’Meara, S.; Latimer, C.; Dicks, E.; Menzies, A.; et al. A systematic, large-scale resequencing screen of X-chromosome coding exons in mental retardation. Nat. Genet. 2009, 41, 535-543. [CrossRef] [PubMed]

28. Kantojärvi, K.; Kotala, I.; Rehnström, K.; Ylisaukko-Oja, T.; Vanhala, R.; Von Wendt, T.N.; Von Wendt, L.; Jarvela, I. Fine mapping of Xq11.1-q21.33 and mutation screening of RPS6KA6, ZNF711, ACSL4, DLG3, and IL1RAPL2 for autism spectrum disorders (ASD). Autism Res. 2011, 4, 228-233. [CrossRef]

29. Van der Werf, I.M.; Van Dijck, A.; Reyniers, E.; Helsmoortel, C.; Kumar, A.; Kalscheuer, V.M.; de Brouwer, A.P.; Kleefstra, T.; van Bokhoven, H.; Mortier, G.; et al. Mutations in two large pedigrees highlight the role of ZNF711 in X-linked intellectual disability. Gene 2017, 605, 92-98. [CrossRef]

30. Ropers, H.-H.; Hamel, B.C.J. X-linked mental retardation. Nat. Rev. Genet. 2005, 6, 46-57. [CrossRef]

31. Wang, Z.-Y.; Leushkin, E.; Liechti, A.; Ovchinnikova, S.; Mößinger, K.; Brüning, T.; Rummel, C.; Grützner, F.; Cardoso-Moreira, M.; Janich, P.; et al. Transcriptome and translatome co-evolution in mammals. Nat. Cell Biol. 2020, 588, 642-647. [CrossRef] [PubMed]

32. Vitezic, M.; Bertin, N.; Andersson, R.; Lipovich, L.; Kawaji, H.; Lassmann, T.; Sandelin, A.; Heutink, P.; Goldowitz, D.; Ha, T.; et al. CAGE-defined promoter regions of the genes implicated in Rett Syndrome. BMC Genom. 2014, 15, 1177. [CrossRef] [PubMed]

33. Zucchelli, S.; Fedele, S.; Vatta, P.; Calligaris, R.; Heutink, P.; Rizzu, P.; Itoh, M.; Persichetti, F.; Santoro, C.; Kawaji, H.; et al. Antisense Transcription in Loci Associated to Hereditary Neurodegenerative Diseases. Mol. Neurobiol. 2019, 56, $5392-5415$. [CrossRef] [PubMed]

34. Kleine-Kohlbrecher, D.; Christensen, J.; Vandamme, J.; Abarrategui, I.; Bak, M.; Tommerup, N.; Shi, X.; Gozani, O.; Rappsilber, J.; Salcini, A.E.; et al. A Functional Link between the Histone Demethylase PHF8 and the Transcription Factor ZNF711 in X-Linked Mental Retardation. Mol. Cell 2010, 38, 165-178. [CrossRef] [PubMed]

35. Kang, H.J.; Kawasawa, Y.I.; Cheng, F.; Zhu, Y.; Xu, X.; Li, M.; Sousa, A.M.M.; Pletikos, M.; Meyer, K.A.; Sedmak, G.; et al. Spatio-temporal transcriptome of the human brain. Nat. Cell Biol. 2011, 478, 483-489. [CrossRef]

36. Fassio, A.; Patry, L.; Congia, S.; Onofri, F.; Piton, A.; Gauthier, J.; Pozzi, D.; Messa, M.; Defranchi, E.; Fadda, M.; et al. SYN1 loss-of-function mutations in autism and partial epilepsy cause impaired synaptic function. Hum. Mol. Genet. 2011, 20, $2297-2307$. [CrossRef] [PubMed]

37. Ming, G.-L.; Song, H. Adult Neurogenesis in the Mammalian Brain: Significant Answers and Significant Questions. Neuron 2011, 70, 687-702. [CrossRef] [PubMed]

38. Melloni, R.; Apostolides, P.; Hamos, J.; DeGennaro, L. Dynamics of synapsin I gene expression during the establishment and restoration of functional synapses in the rat hippocampus. Neuroscience 1994, 58, 683-703. [CrossRef]

39. Tahiliani, M.; Mei, P.; Fang, R.; Leonor, T.; Rutenberg, M.; Shimizu, F.; Li, J.; Rao, A.; Shi, Y. The histone H3K4 demethylase SMCX links REST target genes to X-linked mental retardation. Nat. Cell Biol. 2007, 447, 601-605. [CrossRef] [PubMed]

40. FANTOM Consortium and the RIKEN PMI and CLST; Forrest, A.R.; Kawaji, H.; Rehli, M.; Baillie, J.K.; de Hoon, M.J.; Haberle, V.; Lassmann, T.; Kulakovskiy, I.V.; Lizio, M.; et al. A promoter-level mammalian expression atlas. Nature 2014, 507, 462-470. [CrossRef]

41. Purmann, A.; Toedling, J.; Schueler, M.; Carninci, P.; Lehrach, H.; Hayashizaki, Y.; Huber, W.; Sperling, S. Genomic organization of transcriptomes in mammals: Coregulation and cofunctionality. Genomics 2007, 89, 580-587. [CrossRef] [PubMed]

42. Bienvenu, T.; Poirier, K.; Friocourt, G.; Bahi, N.; Beaumont, D.; Fauchereau, F.; Ben Jeema, L.; Zemni, R.; Vinet, M.-C.; Francis, F.; et al. ARX, a novel Prd-class-homeobox gene highly expressed in the telencephalon, is mutated in X-linked mental retardation. Hum. Mol. Genet. 2002, 11, 981-991. [CrossRef] [PubMed]

43. Curie, A.; Nazir, T.; Brun, A.; Paulignan, Y.; Reboul, A.; Delange, K.; Cheylus, A.; Bertrand, S.; Rochefort, F.; Bussy, G.; et al. The c.429_452 duplication of the ARX gene: A unique developmental-model of limb kinetic apraxia. Orphanet J. Rare Dis. $2014,9,25$. [CrossRef] [PubMed]

44. Jensen, L.R.; Bartenschlager, H.; Rujirabanjerd, S.; Tzschach, A.; Nümann, A.; Janecke, A.R.; Spörle, R.; Stricker, S.; Raynaud, M.; Nelson, J.; et al. A distinctive gene expression fingerprint in mentally retarded male patients reflects disease-causing defects in the histone demethylase KDM5C. PathoGenetics 2010, 3, 2. [CrossRef]

45. Hu, H.; Haas, S.; Chelly, J.; Van Esch, H.; Raynaud, M.; De Brouwer, A.P.M.; Weinert, S.; Froyen, G.; Frints, S.G.; Laumonnier, F.; et al. X-exome sequencing of 405 unresolved families identifies seven novel intellectual disability genes. Mol. Psychiatry 2016, 21, 133-148. [CrossRef] [PubMed]

46. Tzschach, A.; Lenzner, S.; Moser, B.; Reinhardt, R.; Chelly, J.; Fryns, J.-P.; Kleefstra, T.; Raynaud, M.; Turner, G.; Ropers, H.-H.; et al. NovelJARID1C/SMCX mutations in patients with X-linked mental retardation. Hum. Mutat. 2006, 27, 389. [CrossRef] [PubMed]

47. Peng, Y.; Alexov, E. Cofactors-loaded quaternary structure of lysine-specific demethylase 5C (KDM5C) protein: Computational model. Proteins Struct. Funct. Bioinform. 2016, 84, 1797-1809. [CrossRef]

48. Qin, S.; Cho, S.; Chen, T.; Rosenberg-Lee, M.; Geary, D.C.; Menon, V. Hippocampal-neocortical functional reorganization underlies children's cognitive development. Nat. Neurosci. 2014, 17, 1263-1269. [CrossRef] [PubMed]

49. Kouno, T.; Moody, J.; Kwon, A.T.-J.; Shibayama, Y.; Kato, S.; Huang, Y.; Böttcher, M.; Motakis, E.; Mendez, M.; Severin, J.; et al. C1 CAGE detects transcription start sites and enhancer activity at single-cell resolution. Nat. Commun. 2019, 10, 1-12. [CrossRef]

50. Verrillo, L.; Mangano, E.; Drongitis, D.; Merelli, I.; Pischedda, F.; Piccoli, G.; Consolandi, C.; Bordoni, R.; Miano, M.G. A reliable strategy for single-cell RNA sequencing analysis using cryoconserved primary cortical cells. J. Neurosci. Methods 2021, $347,108960$. [CrossRef] [PubMed] 
51. Shoubridge, C.; Tan, M.H.; Fullston, T.; Cloosterman, D.; Coman, D.; McGillivray, G.; Mancini, G.M.; Kleefstra, T.; Gecz, J. Mutations in the nuclear localization sequence of the Aristaless related homeobox; sequestration of mutant ARX with IPO13 disrupts normal subcellular distribution of the transcription factor and retards cell division. PathoGenetics $2010,3,1$. [CrossRef] [PubMed]

52. Yao, Y.; Gao, G.; Liu, K.; Shi, X.; Cheng, M.; Xiong, Y.; Song, S. Projections from D2 Neurons in Different Subregions of Nucleus Accumbens Shell to Ventral Pallidum Play Distinct Roles in Reward and Aversion. Neurosci. Bull. 2021, 37, 623-640. [CrossRef] [PubMed]

53. Poirier, K.; Lacombe, D.; Gilbert-Dussardier, B.; Raynaud, M.; Desportes, V.; De Brouwer, A.P.M.; Moraine, C.; Fryns, J.P.; Ropers, H.H.; Beldjord, C.; et al. Screening of ARX in mental retardation families: Consequences for the strategy of molecular diagnosis. Neurogenetics 2005, 7, 39-46. [CrossRef] [PubMed] 